\title{
BMJ Open Preoperative prediction of potentially preventable morbidity after fast-track hip and knee arthroplasty: a detailed descriptive cohort study
}

Christoffer C Jørgensen, ${ }^{1,2}$ Morten Aa Petersen, ${ }^{3}$ Henrik Kehlet, ${ }^{1,2}$ on behalf of the Lundbeck Foundation Centre for Fast-Track Hip and Knee Replacement Collaborative Group

To cite: Jørgensen CC, Petersen MA, Kehlet $\mathrm{H}$, et al. Preoperative prediction of potentially preventable morbidity after fast-track hip and knee arthroplasty: a detailed descriptive cohort study. BMJ Open 2016;6: e009813. doi:10.1136/ bmjopen-2015-009813

- Prepublication history and additional material is available. To view please visit the journal (http://dx.doi.org/ 10.1136/bmjopen-2015009813).

Received 24 August 2015 Revised 20 October 2015 Accepted 30 November 2015

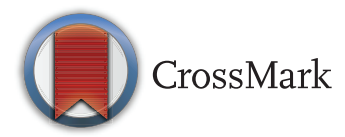

${ }^{1}$ Section for Surgical Pathophysiology, The Juliane Marie Centre, Rigshospitalet, Copenhagen University, Copenhagen, Denmark ${ }^{2}$ The Lundbeck Foundation Centre for Fast-Track Hip and Knee Replacement, Copenhagen, Denmark ${ }^{3}$ The Research Unit, Department of Palliative Medicine, Bispebjerg Hospital, University of Copenhagen, Copenhagen, Denmark

Correspondence to Dr Christoffer Calov Jørgensen; christoffer.calov. joergensen@regionh.dk

\section{ABSTRACT}

Objectives: Construction of a simple preoperative risk score for patients in high risk of potentially preventable 'medical' complications. Secondary objectives were to construct simple preoperative risk scores for 'severe medical', 'surgical' and 'total' potentially preventable complications.

Design: Prospective observational study.

Setting: Elective primary unilateral total hip and knee arthroplasty with prospectively collected preoperative patient characteristics; similar standardised fast-track protocols; evaluation of complications through discharge and medical records; and complete 90 days follow-up through nationwide databases.

Participants: 8373 consecutive unselected total hip arthroplasty (THA) and knee arthroplasty from January 2010 to November 2012.

Results: There were 557 procedures $(6.4 \%)$ followed by potentially preventable complications resulting in hospitalisation $>4$ days or readmission. Of 22 preoperative characteristics, 7 were associated with $379(4.2 \%)$ potentially preventable 'medical' complications. Patients with $\geq 2$ of the following, age $\geq 80$ years, anticoagulant therapy, pulmonary disease, pharmacologically treated psychiatric disorder, anaemia and walking aids, composed $19.1 \%$ of the procedures; $55.7 \%$ constituted potentially preventable 'medical' complications that were mainly falls, mobilisation issues, pneumonias and cardiac arrhythmias. The number needed to be treated for a hypothetical intervention leading to $25 \%$ reduction in potentially preventable 'medical' complications was 34 . THA, use of walking aids and cardiac disease were associated with $189(2.2 \%)$ 'surgical' complications, but no clinically relevant preoperative prediction was possible.

Conclusions: Preoperative identification of patients at high risk of preventable 'medical', but not 'surgical', complications is statistically possible. However, clinical relevance is limited. Future risk indices should differ between 'medical' and 'surgical' complications, and also consider 'preventability' of these.

Trial registration number: NCT01515670.
Strengths and limitations of this study

- A large cohort of patients with total hip arthroplasty and total knee arthroplasty with standardised fast-track protocols and prospective recording on preoperative morbidity.

- Detailed follow-up on postoperative complications through discharge records.

- Differentiation between 'medical' and 'surgical' complications.

- Evaluation of preventability of complications is subjective.

- Validation in other types of fast-track surgery is needed.

\section{INTRODUCTION}

As with any multifactorial problem, prediction of postoperative morbidity in surgical patients is difficult. However, the potential benefit of being able to identify and ultimately allocate high-risk patients to an appropriate level of care is vast. For example, more than $80 \%$ of postoperative deaths have been found to occur in a subgroup of high-risk patients with a likely higher number of nonfatal complications. ${ }^{1}$

Several risk stratification tools have been developed to help clinicians decide whether preoperative optimisation of organ function, changes in perioperative care or postponement of surgery may be indicated. Starting with the American Society of Anesthesiologists' Physical Status score (ASA), ${ }^{2}$ the other scores, such as the Physiological and Operative Severity Score for enUmeration of Mortality and Morbidity (POSSUM),${ }^{3}$ the Surgical APGAR score ${ }^{4}$ and the Charlson Comorbidity Index, ${ }^{5}$ have also been found useful for predicting postoperative morbidity, and may be supplemented by cardiopulmonary exercise tests. ${ }^{6} 7$ 
Recently, internet-based risk calculators published by the American College of Surgeons' National Surgical Quality Improvement Program, have been developed for a variety of procedures and outcomes. ${ }^{8}{ }^{9}$ However, the extent to which these are used in clinical practice is uncertain, and the ideal preoperative risk stratification tool has yet to be found. ${ }^{10}$ Furthermore, if used to guide postoperative care, such a tool should be developed in patients receiving up-to-date evidence-based care and must consider the pathophysiological mechanisms leading to the outcome of interest, thus enabling rational inteventions. ${ }^{11}$

In this context, it must be considered that many risk indices were developed at a time when perioperative care was different from today. Thus, the introduction of enhanced recovery programmes or the so-called 'fasttrack' surgery $^{12}$ has led to changes in surgical, anaesthetic and rehabilitative practices, which have improved postoperative recovery, reduced morbidity and consequently reduced length of hospital stay (LOS) without increasing readmissions. ${ }^{13}$ However, whether traditional perioperative risk assessment is transferable to the concept of fast-track surgery remains to be answered. ${ }^{11}$ In this context, recent studies in fast-track total hip arthroplasty (THA) and total knee arthroplasty (TKA) have found that traditional risk factors, such as age, ${ }^{14} 15$ obesity, ${ }^{16}$ cardiopulmonary disease ${ }^{15}$ smoking or alcohol use, ${ }^{16}{ }^{17}$ and type 2 diabetes, ${ }^{18}$ may have less influence on LOS and postoperative morbidity than has been estimated in previous studies with conventional care. In addition, the types of postoperative morbidity in fasttrack surgery may also differ from previous reports on conventional care. Thus, low incidences of delirium, ${ }^{19}$ venous thromboembolic events ${ }^{20}$ as well as myocardial infarctions and overall mortality have been found in fasttrack THA and TKA. ${ }^{21}$ The combination of different risk profiles and incidences of postoperative complications in fast-track surgery may influence the accuracy of previously established risk indices, thereby calling for procedure-specific studies with implemented fast-track methodology. ${ }^{22}$ Finally, assessment of 'medical' versus 'surgical' complications rather than traditional assessment of 'all cause' morbidity is necessary to define interventional strategies. ${ }^{11}$

The present study attempted to develop a simple numerical score to identify patients at high risk of specific potentially preventable postoperative 'medical' and 'surgical' complications, which is based on previous works on patient-related risk factors and postoperative morbidity in fast-track THA and TKA. ${ }^{15} 1723-25$

\section{METHODS}

The study population was based on a previously published cohort from the Lundbeck Foundation Centre for Fast-Track Hip and Knee Replacement database (LCDB) from January 2010 to November 2012, thus ensuring a high degree of details regarding postoperative morbidity resulting in LOS $>4$ and 90 days readmissions. ${ }^{15} \quad 18 \quad 25$ The cut-off of $>4$ days was chosen based on median LOS being 3 days in 2010. ${ }^{15}$ Eight large Danish Regional/ University hospitals with dedicated arthroplasty departments performing $>400$ procedures/year are currently reporting to the LCDB, which is a prospective registry on preoperative comorbidity registered on ClinicalTrials. gov (ID: NCT01515670). None of the previous investigations based on this cohort have attempted to summarise relevant risk factors into a risk score regarding potentially preventable complications.

All departments have similar fast-track protocols, including spinal anaesthesia, opioid-sparing multimodal analgesia, early mobilisation and with planned discharge to own home. ${ }^{26}$ The data in the LCDB are crossreferenced with the Danish National Patient Registry for information on the index surgery and 90 days follow-up on readmissions and mortality. The Danish National Patient Registry collects data on all admissions in Danish with a data completeness of $>99 \% .{ }^{27}$ Information on preoperative use of continuous anticoagulants, pharmacological antidiabetic and psychiatric treatment is acquired from the Danish National Database on Reimbursed Prescriptions which registers any prescription receiving reimbursement dispensed outside hospitals (the usual practice in the government-funded Danish healthcare system). ${ }^{28}$

LOS was counted as the number of postoperative nights in hospital, including transferrals to other departments. Any case of LOS $>4$ days or readmissions within 90 days were investigated using discharge records to identify the primary cause. This was done by the primary author (CCJ) who consulted the senior author $(\mathrm{HK})$ in case of doubts. If information in the discharge record was deemed insufficient, a review of the entire medical record was performed by CCJ and HK that was followed by discussion until agreement with relation to surgery. In case of death, the complete medical records/ autopsy reports and death certificates were obtained. Only surgery-related readmissions resulting in overnight stay in hospital were considered relevant, that is, uncomplicated urinary tract infections, syncope and acute worsening of chronic obstructive pulmonary disease (COPD) without antibiotic treatment after postoperative day 30 and other admissions obviously unrelated to index surgery (evaluation of newly discovered cancer, eye surgery, cholecystitis, allergic reactions unrelated to postoperative treatment, etc.) were excluded. Causes of LOS $>4$ days and readmissions were subsequently divided into 'surgical' (related directly to the surgical technique and prosthesis components) and 'medical' (related to the surgical procedure or pre-existing conditions but potentially reversible by non-surgical intervention) based on previous work. ${ }^{15}$

Following initial screening for relation to surgery, an evaluation on the potential preventability of the reasons for LOS $>4$ days and readmissions was conducted by both CCJ and HK. This evaluation focused on whether an 
intervention either preoperatively or during the first 2-3 postoperative days could potentially have prevented LOS $>4$ days or readmission. Most 'medical' conditions were included a priori if occurring during primary admission or resulting in readmission $\leq 30$ days after surgery, while some conditions required further evaluation of medical records followed by discussion until mutual agreement was reached by both reviewers. A few conditions, that is, urinary retention $(\mathrm{n}: 12)$ and uncomplicated urinary tract infection (n:18), were excluded due to lack of preoperative information on voiding difficulties, postoperative intermittent catheterisations, and chronic urinary tract infection. Furthermore, postoperative ileus was excluded due to its rarity (n:7), and multifactorial pathogenesis (table 1) 'surgical' readmissions were included $\leq 90$ days after surgery as these often present later than 'medical' complications (table 1). Finally, of the included complications, we classified the following as severe: confirmed cardiac complications, severe hypotension unresponsive to fluids, cerebral stroke or

Table 1 Included and excluded types of complications

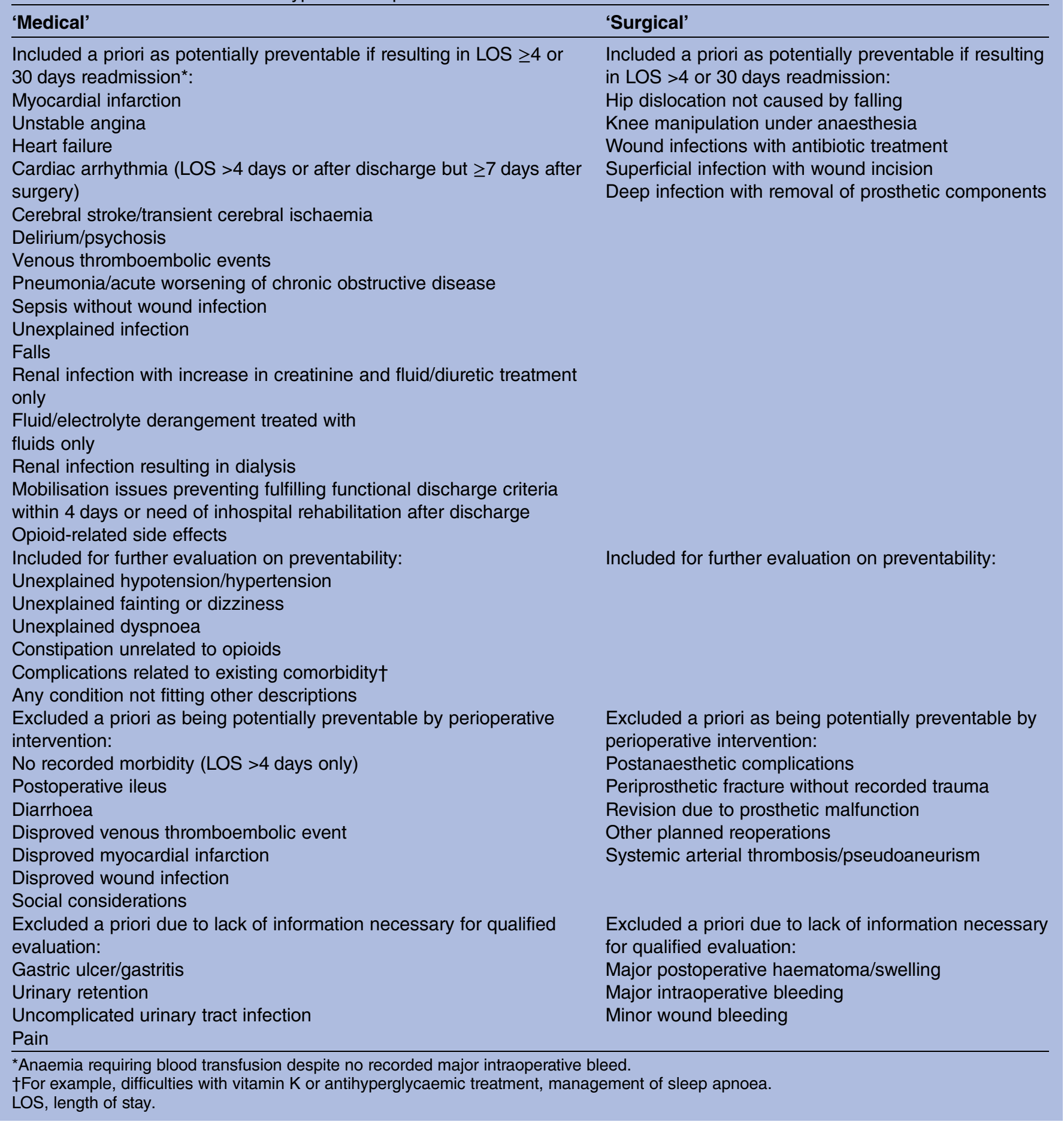


transient cerebral ischaemia, delirium or psychosis, confirmed venous thromboembolic events, pneumonia, worsening of COPD, unexplained dyspnoea with severe hypoxaemia, sepsis, falls, renal affection requiring dialysis, prosthetic infections with surgical interventions, hip dislocations and knee manipulation under anaesthesia (MUA). Only the first potentially preventable 'medical' or 'surgical' complication was included for analysis; however, a subsequent severe complication was included if the first complication did not qualify as such.

\section{Objectives}

Construction of a simple risk score for patients at high risk of potentially preventable 'medical' and 'severe medical' complications leading to LOS $>4$ days or readmission. Secondary outcomes were to construct simple risk scores for 'surgical' and 'total' potentially preventable complications.

\section{Statistics}

Data distribution was analysed using the KolmogorovSmirnov test. Results for non-parametric data are given as medians with IQRs. Risk prediction models for 'total', 'medical', 'severe' medical and 'surgical' complications were constructed using univariate analysis followed by stepwise multiple logistic regression. A significance level $\leq 0.10$ was chosen for univariate analysis to reduce the risk of excluding variables with significant associations after adjustments. ${ }^{29}$ The included variables were: gender, age $\geq 75, \geq 80$ and $\geq 85$ years (cut-offs based on previous work, ${ }^{15}$ with only the most significant age dichotomisations included in the multiple regression), living alone, smoking, alcohol use of $>24 \mathrm{~g}$ /day, use of walking aids, body mass index (BMI) $>30$, surgical procedure (THA vs TKA), hypertension, hypercholesterolaemia, antihyperglycaemic treatment for diabetes, pharmacologically treated psychiatric disorder, ${ }^{25}$ cardiac disease, pulmonary disease, previous cerebral stroke, previous venous thromboembolic event, use of anticoagulants (vitamin $\mathrm{K}$ antagonists, dabigatranetexilate or rivaroxaban). Place of surgery was included in the multiple logistic regression analysis as a random effect. A significance level of 0.05 was chosen for multiple regression models. Two-way interactions between significant variables were added and tested. Receiver operating characteristic of the final model was pictured and area under the curve (AUC) was calculated. To assess whether accuracy could be improved, we used three different weightings for combining the included variables into a total score: one using the ORs, one using a baseline value of 1 for each variable, and one using a value of 1 in case of an OR $<1.90$ and 2 if $\geq 1.90$. The final analysis was done on the largest possible data set, but with minor variations due to missing data for specific preoperative characteristics (table 2).

For the risk score, we calculated the positive (PPV) and negative predictive values (NPV), and the number needed to treat (NNT) with different weighting and

\begin{tabular}{|c|c|}
\hline THA & 4565 (52.2) \\
\hline TKA & $4172(47.8)$ \\
\hline Age/years median (IQR) & $68(67-75)$ \\
\hline$<50$ & $524(6.0)$ \\
\hline $51-60$ & $1465(16.8)$ \\
\hline $61-65$ & $1384(15.8)$ \\
\hline $66-70$ & $1717(19.7)$ \\
\hline $71-75$ & $1546(17,7)$ \\
\hline $76-80$ & $1191(13.6)$ \\
\hline $81-85$ & $650(7.4)$ \\
\hline$>85$ & $260(3.0)$ \\
\hline Females & 5031 (57.8) \\
\hline BMI median (IQR) & $27.7(24.8-31.2)$ \\
\hline$<18.5$ & $69(0.8)$ \\
\hline $18.5-24.9$ & $2284(26.2)$ \\
\hline 25.0-29.9 & 3475 (39.9) \\
\hline $30-34.9$ & $1960(22.5)$ \\
\hline $35-39.9$ & 685 (7.9) \\
\hline$>40$ & $237(2.7)$ \\
\hline Missing & $27(0.3)$ \\
\hline $\mathrm{BMI} \geq 30$ & $2882(33.0)$ \\
\hline Missing & $27(0.3)$ \\
\hline Anaemia & $1107(12.8)$ \\
\hline Missing & $77(0.9)$ \\
\hline Walking aids & $2126(24.8)$ \\
\hline Missing & $177(2.0)$ \\
\hline Living alone & 2954 (33.8) \\
\hline With others & $5783(66.2)$ \\
\hline Missing & $0(0.0)$ \\
\hline Smoking & 1325 (15.3) \\
\hline Missing & $90(1.0)$ \\
\hline Alcohol >24 g/day & 599 (6.9) \\
\hline Missing & $73(0.8)$ \\
\hline Hypertension & $4585(52.8)$ \\
\hline Missing & $55(0.6)$ \\
\hline Hypercholesterolaemia & $2572(29.4)$ \\
\hline Missing & $80(0.9)$ \\
\hline $\begin{array}{l}\text { Diabetes with antihyperglycaemic } \\
\text { treatment }\end{array}$ & 861 (9.9) \\
\hline Missing & $35(0.4)$ \\
\hline Anticoagulant treatment ${ }^{*}$ & $445(5.1)$ \\
\hline Missing & $0(0.0)$ \\
\hline Cardiac disease & 1083 (12.4) \\
\hline Missing & $128(1.5)$ \\
\hline Pulmonary disease & $658(7.6)$ \\
\hline Missing & $73(0.8)$ \\
\hline PsD with pharmacological treatment & $975(11.2)$ \\
\hline Missing & $393(4.5)$ \\
\hline Previous stroke & $528(6.1)$ \\
\hline Missing & $129(1.5)$ \\
\hline Previous venous thromboembolic event & $469(5.5)$ \\
\hline Missing & $152(1.7)$ \\
\hline
\end{tabular}

chose combinations of the included variables to find a clinically relevant cut-off for 'high-risk' patients. We also calculated how many complications would have been 
avoided in our study cohort if applying hypothetical interventions reducing complications by $50 \%, 33 \%$ and $25 \%$. When testing differences in occurrences of specific complications, we used a significance level of 0.001 to account for multiple comparisons. Analysis was done using SPSS V.20 ((IBM Corporation, Armonk, New York, USA) and SAS V.9.3 (SAS institute inc, Cary, North Carolina, USA) )

\section{RESULTS}

A total of 8804 procedures in 8288 patients were eligible for analysis. We excluded $67(0.8 \%)$ procedures in patients coming from nursing homes or other institutions, as these composed a very small and potentially different patient population. Of the remaining 8737 procedures, $1362(15.6 \%)$ were followed by complications leading to either LOS $>4$ days or readmissions, but only 568 complications $(6.4 \%$ of all procedures, range per year: $5.8-7.0 \%$ ) were potentially preventable). Of 379 (4.2\%) 'medical' complications of which 213 (2.3\%) were 'severe', $258(3.0 \%)$ resulted in LOS $>4$ days and $121(1.4 \%)$ resulted in readmissions. Correspondingly, of 189 (2.2\%) 'surgical' complications of which 177 (2.0\%) were 'severe', $13(0.1 \%)$ and $176(2.0 \%)$ resulted in LOS >4 days and readmissions, respectively (see online table supplementary data 1 for details).

\section{Primary objectives: 'medical' and 'severe medical' complications}

The final regression model for 'medical' complications included the following seven characteristics: age $\geq 80$ years, hypertension, use of anticoagulants, pulmonary disease, pharmacologically treated psychiatric disorder, anaemia and use of walking aids (see online supplementary data 2 table S1 for results of logistic regression). The AUC was between 0.75 and 0.76 regardless of weighting, and with an estimated risk of developing a 'medical' complication leading to either LOS $>4$ days or readmission of $67 \%$ with seven preoperative predictors. No patient had seven predictors and only 6 $(0.07 \%)$ patients had six. All combinations of predictors had a NPV of $\geq 0.96$, but the highest PPV was only 0.32 ( $\geq 5$ predictors); these patients were only $0.9 \%$ of the population (table 3). Assigning a value of 1 for each predictor and using a cut-off of $\geq 3$ selected $14.2 \%$ of patients with a $12.9 \%$ complication rate and encompassing $45.0 \%$ of all 'medical' complications. If excluding hypertension, which was present in $52.4 \%$ of the population, from the relevant medical predictors (medical predictors excluding hypertension, MPEH) and using a cut-off of $\geq 2,19.1 \%$ (range13.9-21.2\% per department) of patients would be risk patients (table 3 figure 1) encompassing $55.7 \%$ (range $50.0-65.7 \%$ per department) of 'medical' complications. The NNT with a hypothetical intervention reducing 'medical' complications by $25 \%$ in patients with $\geq 2$ MPEH was 34 , and 46 (14.0\%) complications would have been avoided in the cohort. No improvement in predictive ability was found when assigning a value of 2 to predictors with an OR of $\geq 1.9$ (maximum of 12 ), regardless of excluding hypertension (table 3).

When analysing the cases of 'severe medical' complications, we found that anaemia and hypertension no longer qualified as predictors (see online supplementary data 2 table S2 for results of logistic regression). AUC was 0.75 and the estimated risk was $45.1 \%$ with all five predictors. No patient had all predictors and only $0.3 \%$ had 4. Using a cut-off of $\geq 2$ of these five predictors would result in $14.9 \%$ risk patients, who contributed $48.4 \%$ of all severe complications. However, if using $\geq 2$ MPEH as cut-off, $54.2 \%$ of the severe complications occurred in risk patients (table 3 and figure 1), resulting in a NNT of 59 and avoiding $26(14.0 \%)$ complications.

Overall, the most frequent complications in patients with $\geq 2$ MPEH were: anaemia, mobilisation issues, pulmonary, cardiac, renal/fluid related and falls. In contrast, there were few cases of opioid-related morbidity, delirium, thromboembolic events and severe renal complications (figure 2A,B). Of 'severe' complications, the significantly more were pulmonary, cardiac and due to falls in patients with $\geq 2$ MPEH versus patients with $<2$ MPEH ( $<<0.0001$; figure 3 ). The majority of pulmonary and cardiac complications were pneumonias (n: 23) and arrhythmias (n:18) occurring in $1.5 \%$ and $1.2 \%$ of patients, respectively, with $\geq 2 \mathrm{MPEH}$.

\section{Secondary objectives: 'surgical' and 'total' complications}

For 'surgical' complications, the final regression model included three characteristics: joint of surgery, use of walking aids and cardiac disease. As only three characteristics, one of which was the surgical procedure, were associated with 'surgical' morbidity no clinically relevant prediction based on preoperative risk factors was possible. However, there were more hip dislocations in patients with THA $(3.6 \%$ vs $1.5 \%, \mathrm{p}<0.001)$ and no MUAs in patients with TKA $(0.0 \%$ vs $0.9 \%, \mathrm{p}=0.006)$ with $\geq 2$ MPEH. For details on analysis on 'surgical' complications see supplementary data 3. Multiple logistic regression analysis on 'total' complications found a significant association with the following preoperative characteristics: age $\geq 75$ years, hypertension, cardiac disease, pulmonary disease, pharmacologically treated psychiatric disorder, anaemia and use of walking aids. The risk of developing a complication leading to either LOS $>4$ days or readmission was $49.8 \%$ if all seven predictors were present prior to surgery; however, no patient had all seven predictors and only $36(0.5 \%)$ patients had six. For details of analysis on 'total' complications see supplementary data 4 .

\section{Mortality}

Sixteen $(0.2 \%)$ deaths occurred within 30 days after surgery. Of these, one was unrelated to surgery (cancer related) and one was in a patient with some missing preoperative data (pulmonary embolism). Of the 14 
Table 3 Predictive ability of combinations of significant predictors

\begin{tabular}{|c|c|c|c|c|c|c|c|c|c|c|c|c|c|c|c|}
\hline \multirow{2}{*}{ Outcome } & \multirow[b]{2}{*}{ Characteristic (\%) } & \multicolumn{2}{|l|}{ Events (\%) } & \multirow{2}{*}{$\begin{array}{l}\text { Percentage } \\
\text { of events }\end{array}$} & \multirow{2}{*}{$\begin{array}{l}\text { Percentage } \\
\text { correct }\end{array}$} & \multirow[b]{2}{*}{ Sensitivity } & \multirow[b]{2}{*}{ Specificity } & \multirow[b]{2}{*}{ PPV } & \multirow[b]{2}{*}{ NPV } & \multirow{2}{*}{$\begin{array}{l}\text { NNT } \\
50\end{array}$} & \multirow{2}{*}{$\begin{array}{l}\text { NNT } \\
33\end{array}$} & \multirow{2}{*}{$\begin{array}{l}\text { NNT } \\
25\end{array}$} & \multirow{2}{*}{$\begin{array}{l}\mathrm{AE} \\
50\end{array}$} & \multirow{2}{*}{$\begin{array}{l}\mathrm{AE} \\
33\end{array}$} & \multirow{2}{*}{$\begin{array}{l}\text { AE } \\
25 \\
\end{array}$} \\
\hline & & Yes & No & & & & & & & & & & & & \\
\hline \multirow{14}{*}{$\begin{array}{l}\text { 'Medical' } \\
\text { complications } \\
\text { n: } 327\end{array}$} & Age $\geq 80$ years $(12.3)$ & $118(11.9)$ & 872 & 36.1 & 86.5 & 36.09 & 88.67 & 0.12 & 0.97 & 17 & 25 & 34 & 59 & 39 & 30 \\
\hline & Walking aids (24.2) & $187(9.6)$ & 1753 & 57.2 & 76.4 & 57.19 & 77.22 & 0.10 & 0.98 & 21 & 31 & 41 & 94 & 62 & 47 \\
\hline & Hypertension (52.4) & $221(5.3)$ & 3982 & 67.6 & 49.0 & 67.58 & 48.25 & 0.05 & 0.97 & 38 & 57 & 76 & 111 & 74 & 55 \\
\hline & Anticoagulants (5.0) & $42(10.5)$ & 357 & 12.8 & 92.0 & 12.84 & 95.36 & 0.11 & 0.96 & 19 & 29 & 38 & 21 & 14 & 11 \\
\hline & Anaemia (12.4) & $94(9.4)$ & 904 & 28.7 & 85.8 & 28.75 & 88.25 & 0.09 & 0.97 & 21 & 32 & 42 & 47 & 31 & 24 \\
\hline & Psychological disorder (11.6) & $78(8.4)$ & 851 & 23.9 & 86.3 & 23.85 & 88.94 & 0.08 & 0.96 & 24 & 36 & 48 & 39 & 26 & 20 \\
\hline & Pulmonary disease (7.4) & $46(7.7)$ & 551 & 14.1 & 89.6 & 14.07 & 92.84 & 0.08 & 0.96 & 26 & 39 & 52 & 23 & 15 & 12 \\
\hline & $\geq 3$ predictors $(14.3)$ & $147(12.9)$ & 996 & 45.0 & 85.3 & 44.95 & 87.05 & 0.13 & 0.97 & 16 & 23 & 31 & 74 & 49 & 37 \\
\hline & $\geq 4$ predictors (4.3) & $68(19.8)$ & 275 & 20.8 & 93.3 & 20.80 & 96.43 & 0.20 & 0.97 & 10 & 15 & 20 & 34 & 23 & 17 \\
\hline & $\geq 5$ predictors $(0.9)$ & $24(32.4)$ & 50 & 7.3 & 95.6 & 7.34 & 99.35 & 0.32 & 0.96 & 6 & 9 & 12 & 12 & 8 & 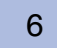 \\
\hline & $\geq 4$ MP2 (18.1) & $174(12.0)$ & 1276 & 53.2 & 82.2 & 53.21 & 83.42 & 0.12 & 0.98 & 17 & 25 & 33 & 87 & 58 & 44 \\
\hline & $\geq 5$ MP2 (12.8) & $139(13.6)$ & 886 & 42.5 & 86.6 & 42.51 & 88.48 & 0.14 & 0.97 & 15 & 22 & 29 & 70 & 46 & 35 \\
\hline & $\geq 2$ MPEH (19.1) & $182(11.9)$ & 1348 & 55.7 & 81.4 & 55.66 & 82.50 & 0.12 & 0.98 & 17 & 25 & 34 & 91 & 61 & 46 \\
\hline & $\geq 3 \mathrm{MPEH} 2$ (5.6) & $83(18.5)$ & 366 & 55.7 & 92.4 & 25.38 & 95.14 & 0.18 & 0.97 & 11 & 16 & 22 & 42 & 28 & 21 \\
\hline \multirow{11}{*}{$\begin{array}{l}\text { 'Severe medical' } \\
\text { complications } \\
n: 213\end{array}$} & Age $\geq 80$ years $(12.3)$ & $70(7.0)$ & 928 & 36.8 & 87.1 & 36.84 & 88.32 & 0.07 & 0.98 & 29 & 43 & 57 & 35 & 23 & 18 \\
\hline & Walking aids (24.2) & $108(5.5)$ & 1861 & 56.8 & 76.1 & 56.84 & 76.57 & 0.05 & 0.99 & 36 & 55 & 73 & 54 & 36 & 27 \\
\hline & Anticoagulants (4.9) & $27(6.7)$ & 378 & 14.2 & 93.3 & 14.21 & 95.24 & 0.07 & 0.98 & 30 & 45 & 60 & 14 & 9 & 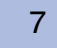 \\
\hline & Psychological disorder (11.6) & $48(5.1)$ & 895 & 25.3 & 87.2 & 25.26 & 88.73 & 0.05 & 0.98 & 39 & 59 & 79 & 24 & 16 & 12 \\
\hline & Pulmonary disease (7.5) & $31(5.11)$ & 577 & 16.3 & 90.9 & 16.32 & 92.73 & 0.05 & 0.98 & 39 & 59 & 78 & 16 & 10 & \\
\hline & $\geq 2$ predictors $(14.9)$ & $92(7.6)$ & 1122 & 48.4 & 85.0 & 48.42 & 85.87 & 0.08 & 0.99 & 26 & 40 & 53 & 46 & 31 & 23 \\
\hline & $\geq 3$ predictors $(3.1)$ & $31(12.4)$ & 218 & 16.3 & 95.4 & 16.32 & 97.26 & 0.12 & 0.98 & 16 & 24 & 32 & 16 & 10 & \\
\hline & $\geq 4$ predictors $(0.3)$ & $6(23.1)$ & 20 & 3.2 & 97.5 & 3.16 & 99.75 & 0.23 & 0.98 & 9 & 13 & 17 & 3 & 2 & \\
\hline & $\geq 3 \mathrm{MP}(14.3)$ & $83(7.3)$ & 1060 & 43.7 & 85.5 & 44.62 & 86.47 & 0.07 & 0.99 & 28 & 41 & 55 & 42 & 28 & 21 \\
\hline & $\geq 4$ MP2 (18.1) & $98(7.0)$ & 1352 & 51.6 & 82.1 & 52.69 & 82.74 & 0.07 & 0.99 & 30 & 44 & 59 & 49 & 33 & 25 \\
\hline & $\geq 2$ MPEH (19.1) & $103(6.7)$ & 1427 & 54.2 & 81.2 & 55.38 & 81.79 & 0.07 & 0.99 & 30 & 45 & 59 & 52 & 34 & 26 \\
\hline
\end{tabular}

Number of included patients is 8021 for 'medical' complications and 8132 for 'severe medical' complications due to $8.1 \%$ and $6.9 \%$ with missing data on relevant predictors.

$\mathrm{AE} \mathrm{25}$, avoided events in the cohort with $25 \%$ event reduction; AE 30 , avoided events in the cohort with $30 \%$ event reduction; AE 50 , avoided events in the cohort with $50 \%$ event reduction

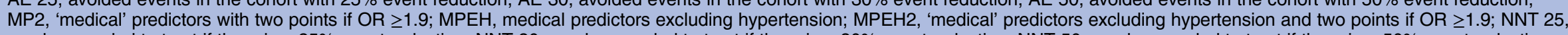
number needed to treat if there is a $25 \%$ event reduction; NNT 30 , number needed to treat if there is a $30 \%$ event reduction; NNT 50 , number needed to treat if there is a $50 \%$ event reduction;

NPN, negative predictive value; PPV, positive predictive value. 
Figure 1 Relationship between per cent of complications and percentage of population for weightings. MPEH, medical predictors excluding hypertension. combinations of predictors and

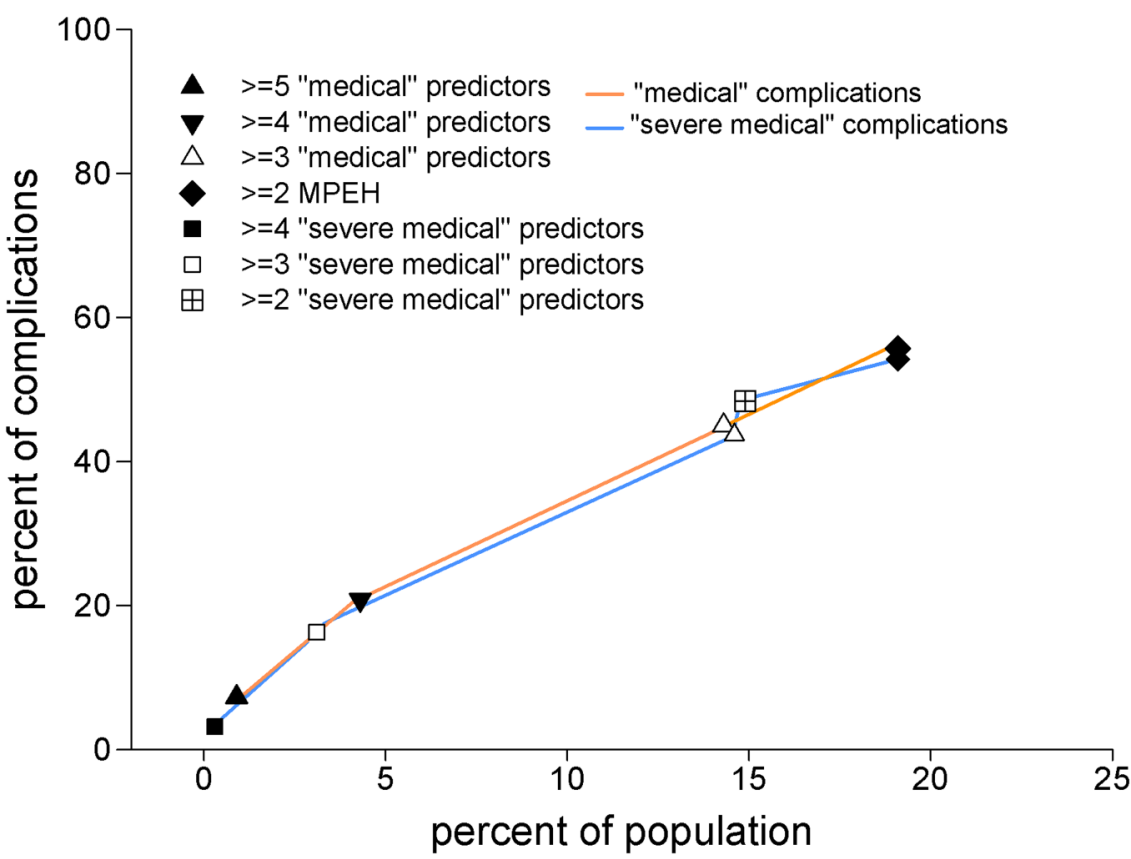

potentially $(0.1 \%)$ surgery-related deaths, $11(0.7 \%)$ were in patients with $\geq 2$ MPEH versus $3(0.04 \%)$ with $<2$ MPEH $(\mathrm{p}<0.001)$.

\section{DISCUSSION}

Predicting which patients will develop complications after surgery is difficult, but focus on improving postoperative outcomes through better perioperative care is increasing. ${ }^{13} 3031$ Our study in fast-track THA and TKA found that although identification of high-risk patients for potentially preventable 'medical' complications based on preoperative risk factors is statistically possible, the clinical relevance is limited by diverse types of complications and a high NNT. Furthermore, we found that predictors for 'surgical' and 'medical' complications were not the same, thus demonstrating the importance of distinguishing between these, especially considering that neither statistically nor clinically relevant preoperative prediction was possible regarding 'surgical' complications. Finally, our study demonstrated the necessity of considering preventability of complications, as this was the case in only about $40 \%$ of patients with LOS $>4$ days or readmissions. Similar results have been reported in mixed patients, where the fraction of preventable unplanned readmissions is about $20-30 \% .{ }^{32} 33$

Apart from the obstacles of few potentially "preventable' complications and different predictors of potentially preventable 'medical' and 'surgical' complications, there is the problem of defining suitable strategies for

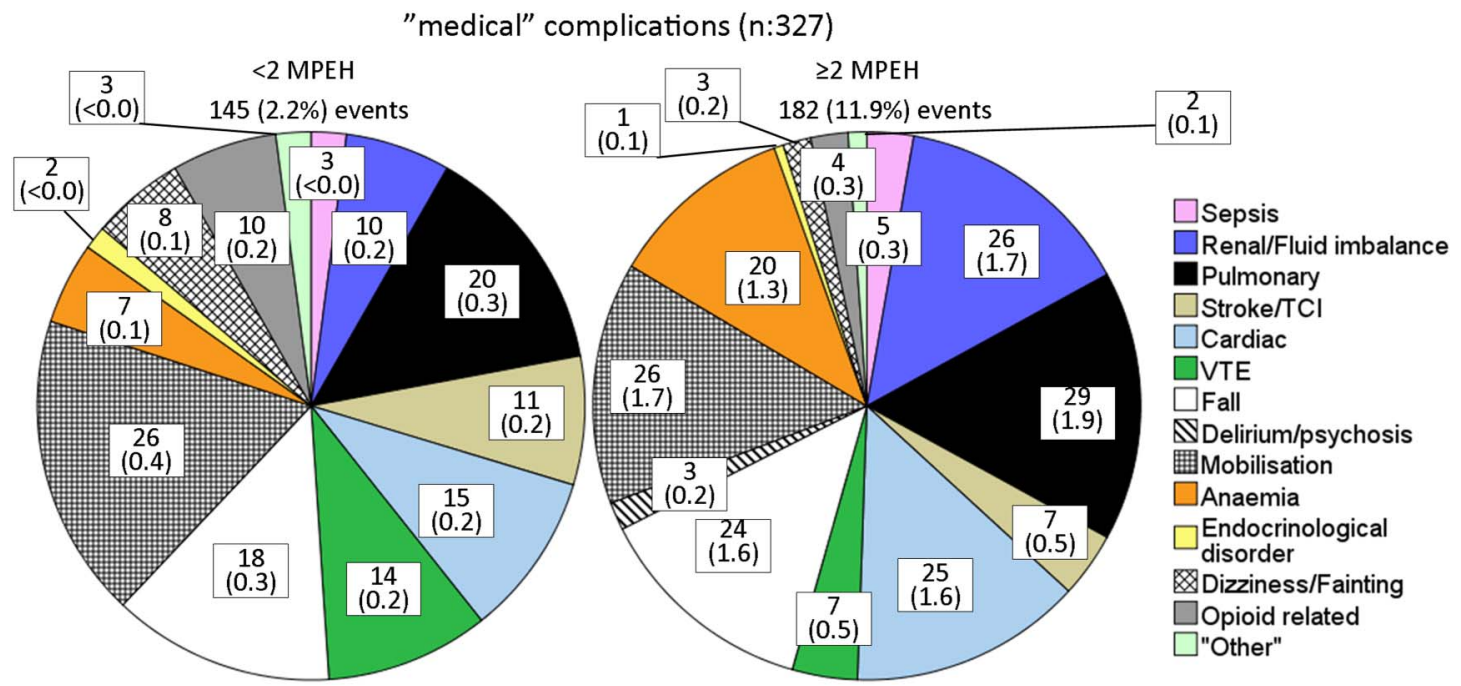

Figure 2 Occurrence and fraction of specific types of 'medical' complications in patients with <2 'medical' predictors excluding hypertension (MPEH) n: 6491 vs $\geq 2 \mathrm{MPEH}$ n: 1530 . TCl, transient cerebral ischaemia; VTE, venous thromboembolic event. 
"severe medical" complications (n:186)

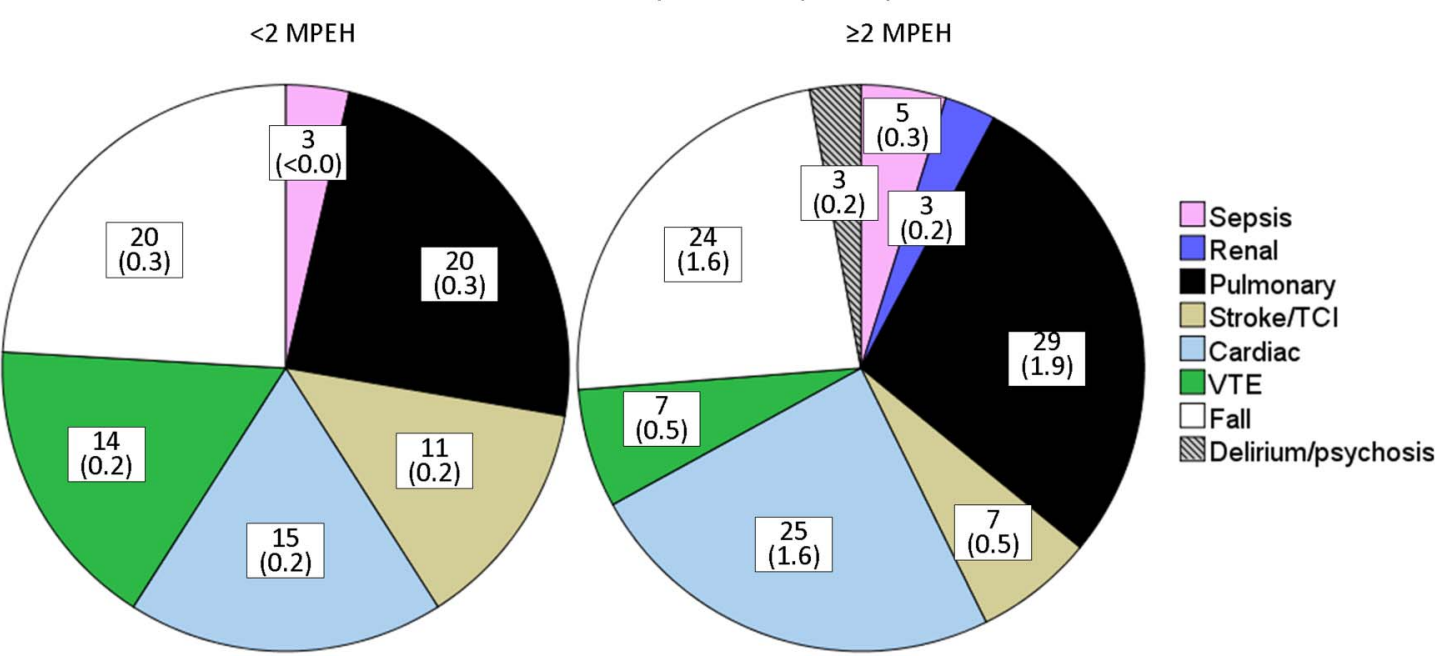

Figure 3 Occurrence and fraction of specific types of 'severe medical' complications in patients with $<2$ 'medical' predictors excluding hypertension (MPEH) n: 6491 vs $\geq 2$ MPEH n: 1530. TCl, transient cerebral ischaemia; VTE, venous thromboembolic event.

reducing these. Thus, 'medical' complications may be reduced by further optimisation of the fast-track methodology, while 'surgical' complications would be dependent on surgical expertise. ${ }^{11}$

In our opinion, the most clinically relevant cut-off for defining patients at high risk of potentially preventable 'medical' complications was $\geq 2 \mathrm{MPEH}$. This encompassed about $20 \%$ of all patients, with minor variation between departments, and these accounted for more than half of the 'medical' complications. Interventional studies targeting patients with $\geq 2$ MPEH could focus on falls, mobilisation issues, pneumonia and cardiac arrhythmia as these occurred most frequently. Unfortunately, a significant reduction of such diverse postoperative 'medical' complications is likely to require a multimodal interventional strategy, including correction of preoperative anaemia and ward rounds by anaesthesiologists or geriatricians. Such attempts have shown promising results both in orthopaedic and in other types of surgery. ${ }^{34-36}$ However, designing the required randomised trials within a standardised fast-track protocol will be challenging, especially considering the large number of patients needed.

Prediction of 'surgical' complications was all but impossible in the present study, but it is interesting that no cases of MUA were present in the patients with $\geq 2$ MPEH, supporting our previous findings of MUA being associated with younger age and independence from walking aids. ${ }^{37}$ Finally, how to reduce the number of hip dislocations in patients with $\geq 2$ MPEH deserves further study.

Previous studies of smaller cohorts contributing to the present study $^{15} \quad 17 \quad 18 \quad 20$ and in other study cohorts $^{14161938}$ have found a reduced importance of conventional risk factors and decreased postoperative morbidity in fast-track THA and TKA compared with studies with conventional perioperative care. This was confirmed in the present study where smoking, alcohol use, diabetes requiring antihyperglycaemic treatment and a BMI of $>30$ were not associated with postoperative complications, and even when using $\geq 2$ MPEH to define patients at risk of 'medical' complications, the high NNT limited the clinical relevance. However, whether this also applies to other types of fast-track surgery and to other patient populations remains unknown. Thus, characterisation of the high-risk patients and postoperative morbidity in other fast-track surgical procedures and populations is needed. This may be especially important regarding obesity, where it could be argued that our BMI cut-off should have been placed at 35 or beyond. ${ }^{39}$

Our study is subject to some limitations. As it was observational, we can only provide associations. However, the intention was to define a clinically relevant group of high-risk patients, and not to demonstrate causality between preoperative characteristics and specific complications. Furthermore, evaluation of complications is subjective and the inclusion of three or more reviewers to solve potential disagreements by majority vote may have been preferable. It could also be argued that we should have used an existing index for evaluation of postoperative complications. Unfortunately, existing indices are subject to limitations, for example, the Dindo-Clavien index focuses on the most severe complications, with no distinction between 'medical' or 'surgical' morbidity and without consideration of preoperative risk factors. ${ }^{40}$ The Comprehensive Complication Index expresses all complications as a single score,${ }^{41}$ but does not consider the order of occurrence or whether the initial complications led to subsequent complications. The Postoperative Morbidity Survey ${ }^{42}$ may be inadequate for recording morbidity in fast-track orthopaedic patients. A weakness from evaluating patient records is that unrecorded information may have led to misclassification regarding the preventability 
of the complication and whether it was 'medical' or 'surgical'. Nonetheless, this approach is likely to be superior to using diagnostic codes, and has been proposed as the best way of determining preventability of readmissions. ${ }^{43}$ Finally, the results should ideally be confirmed in a different cohort having fast-track THA and TKA to confirm the validity and predictive ability of the identified risk factors.

The strengths of our study include a detailed analysis of postoperative morbidity distinguishing between what came first; whether 'medical' or 'surgical', it made use of prospectively recorded data on patient characteristics, and had a well-described standardised fast-track setup ${ }^{26}$ with consecutive unselected patients and nationwide databases with complete follow-up. ${ }^{27} 28$

In conclusion, this study demonstrates the importance of distinguishing between 'medical' and 'surgical' morbidity when investigating the role of preoperative risk factors. Additionally, the predictive value of the six relevant predictors for developing potentially preventable 'medical' complications was limited and no clinically relevant prediction of 'surgical' complications was possible.

Collaborators The members of the Lundbeck Foundation Center for Fast-track Hip and Knee Replacement Collaborative Group helped implement the fast-track protocols and the LCDB questionnaire at their respective departments, and revised the manuscript: Kjeld Soeballe DmSci, Department of Orthopaedics, Aarhus University Hospital, Aarhus University, Denmark. Torben B Hansen DMSci, Department of Orthopaedics, Regional Hospital Holstebro, Aarhus University, Denmark. Henrik Husted DMSci, Orthopaedic Department, Hvidovre University Hospital, Copenhagen University, Denmark. Mogens B Laursen PhD, Orthopaedic Division, Aalborg University Hospital, Aalborg University, Denmark. Lars T Hansen MD, Orthopaedic Department, Sydvestjysk Hospital Esbjerg/Grindsted, Denmark. Per Kjærsgaard-Andersen MD, Department of Orthopaedics, Vejle Hospital, University of Southern Denmark, Denmark. Søren Solgaard DMSci, Department of Orthopaedics, Gentofte University Hospital, Denmark.

Contributors CCJ contributed to the idea and study design, collection, evaluation and analysis of data, writing and revising the primary draft. MAP contributed to the refinement of the statistical methods, statistical analysis and revision of the primary draft. HK contributed to the idea and study design, evaluation and analysis of data, and revision of the primary draft.

Funding The study was supported by the Lundbeck Foundation, Copenhagen, Denmark, grant (R25-A2702). The Lundbeck Foundation is independent from the Lundbeck Pharmaceutical Company and had no influence on any part of the study design or preparation of the manuscript.

Competing interests HK is a board member of the Health Care initiative Biomet Rapid Recovery.

Ethics approval This study required no approval from the Regional Ethics committee and permission was granted from the Danish National Board of Health (j.nr:3-3013-56/1/HKR), and the Danish Data Protection Agency (j.nr: 20047-58-0015) to review and store medical records without informed consent.

Provenance and peer review Not commissioned; externally peer reviewed.

Data sharing statement Data set is available from the corresponding author.

Open Access This is an Open Access article distributed in accordance with the Creative Commons Attribution Non Commercial (CC BY-NC 4.0) license, which permits others to distribute, remix, adapt, build upon this work noncommercially, and license their derivative works on different terms, provided the original work is properly cited and the use is non-commercial. See: http:// creativecommons.org/licenses/by-nc/4.0/

\section{REFERENCES}

1. Pearse RM, Harrison DA, James $P$, et al. Identification and characterisation of the high-risk surgical population in the United Kingdom. Crit Care 2006;10:R81.

2. Keats AS. The ASA classification of physical status-a recapitulation. Anesthesiology 1978;49:233-6.

3. Copeland GP, Jones D, Walters M. POSSUM: a scoring system for surgical audit. Br J Surg 1991;78:355-60.

4. Reynolds $P Q$, Sanders NW, Schildcrout JS, et al. Expansion of the surgical Apgar score across all surgical subspecialties as a means to predict postoperative mortality. Anesthesiology 2011;114: 1305-12.

5. Charlson ME, Pompei P, Ales KL, et al. A new method of classifying prognostic comorbidity in longitudinal studies: development and validation. J Chronic Dis 1987;40:373-83.

6. West MA, Lythgoe D, Barben CP, et al. Cardiopulmonary exercise variables are associated with postoperative morbidity after major colonic surgery: a prospective blinded observational study. Br J Anaesth 2014;112:665-71.

7. Grant SW, Hickey GL, Wisely NA, et al. Cardiopulmonary exercise testing and survival after elective abdominal aortic aneurysm repairdagger. Br J Anaesth 2015;114:430-6.

8. Bilimoria KY, Liu Y, Paruch JL, et al. Development and evaluation of the universal ACS NSQIP surgical risk calculator: a decision aid and informed consent tool for patients and surgeons. J Am Coll Surg 2013;217:833-42.

9. Hyder JA, Wakeam E, Habermann EB, et al. Derivation and validation of a simple calculator to predict home discharge after surgery. J Am Coll Surg 2014;218:226-36.

10. Moonesinghe SR, Mythen MG, Das $P$, et al. Risk stratification tools for predicting morbidity and mortality in adult patients undergoing major surgery: qualitative systematic review. Anesthesiology 2013;119:959-81.

11. Kehlet $\mathrm{H}$, Mythen $\mathrm{M}$. Why is the surgical high-risk patient still at risk? Br J Anaesth 2011;106:289-91.

12. Kehlet $\mathrm{H}$, Wilmore DW. Evidence-based surgical care and the evolution of fast-track surgery. Ann Surg 2008;248:189-98.

13. Kehlet $\mathrm{H}$. Fast-track hip and knee arthroplasty. Lancet 2013;381:1600-2.

14. Starks I, Wainwright TW, Lewis J, et al. Older patients have the most to gain from orthopaedic enhanced recovery programmes. Age Ageing 2014;43:642-8.

15. Jorgensen $\mathrm{CC}$, Kehlet $\mathrm{H}$. Role of patient characteristics for fast-track hip and knee arthroplasty. Br J Anaesth 2013;110:972-80.

16. den Hartog YM, Mathijssen NM, Hannink G, et al. Which patient characteristics influence length of hospital stay after primary total hip arthroplasty in a 'fast-track' setting? Bone Joint J 2015;97-B:19-23.

17. Jorgensen CC, Kehlet $\mathrm{H}$. Outcomes in smokers and alcohol users after fast-track hip and knee arthroplasty. Acta Anaesthesiol Scand 2013;57:631-8.

18. Jorgensen CC, Madsbad S, Kehlet H. Postoperative morbidity and mortality in type-2 diabetics after fast-track primary total hip and knee arthroplasty. Anesth Analg 2015;120:230-8.

19. Krenk L, Rasmussen LS, Hansen TB, et al. Delirium after fast-track hip and knee arthroplasty. Br J Anaesth 2012;108:607-11.

20. Jorgensen CC, Jacobsen MK, Soeballe K, et al. Thromboprophylaxis only during hospitalisation in fast-track hip and knee arthroplasty, a prospective cohort study. BMJ Open 2013;3: e003965.

21. Savaridas T, Serrano-Pedraza I, Khan SK, et al. Reduced medium-term mortality following primary total hip and knee arthroplasty with an enhanced recovery program. A study of 4,500 consecutive procedures. Acta Orthop 2013;84:40-3.

22. Kehlet $\mathrm{H}$, Jorgensen $\mathrm{CC}$. Predicting postoperative morbidity: in what procedures and what patients? Anesthesiology 2014;120:1297.

23. Jans $\mathrm{O}$, Jorgensen $\mathrm{C}$, Kehlet $\mathrm{H}$, et al. Role of preoperative anemia for risk of transfusion and postoperative morbidity in fast-track hip and knee arthroplasty. Transfusion 2014;54:717-26.

24. Jorgensen $\mathrm{CC}$, Kehlet $\mathrm{H}$. Fall-related admissions after fast-track total hip and knee arthroplasty-cause of concern or consequence of success? Clin Interv Aging 2013;8:1569-77.

25. Jorgensen CC, Knop J, Nordentoft M, et al. Psychiatric disorders and psychopharmacologic treatment as risk factors in elective fast-track total hip and knee arthroplasty. Anesthesiology 2015;123:1281-91.

26. Husted $\mathrm{H}$, Solgaard S, Hansen TB, et al. Care principles at four fast-track arthroplasty departments in Denmark. Dan Med Bull 2010;57:A4166.

27. Andersen TF, Madsen $\mathrm{M}$, Jorgensen $\mathrm{J}$, et al. The Danish National Hospital Register. A valuable source of data for modern health sciences. Dan Med Bull 1999;46:263-8. 
28. Johannesdottir SA, Horváth-Puhó E, Schmidt M, et al. Existing data sources for clinical epidemiology: the Danish National Database of Reimbursed Prescriptions. Clin Epidemiol 2012;4:1-11.

29. Hosmer DW, Lemenshow S. Applied logistic regression. 2nd edn. New York: Wiley, Inc, 2000.

30. Cannesson M, Ani F, Mythen MM, et al. Anaesthesiology and perioperative medicine around the world: different names, same goals. Br J Anaesth 2015;114:8-9.

31. Grocott MP, Pearse RM. Perioperative medicine: the future of anaesthesia? Br J Anaesth 2012;108:723-6.

32. van Walraven $C$, Jennings A, Forster AJ. A meta-analysis of hospital 30-day avoidable readmission rates. J Eval Clin Pract 2012:18:1211-18.

33. Brown EG, Burgess D, Li CS, et al. Hospital readmissions: necessary evil or preventable target for quality improvement. Ann Surg 2014;260:583-9.

34. Harari D, Hopper A, Dhesi J, et al. Proactive care of older people undergoing surgery ('POPS'): designing, embedding, evaluating and funding a comprehensive geriatric assessment service for older elective surgical patients. Age Ageing 2007;36:190-6.

35. Foss NB, Christensen DS, Krasheninnikoff M, et al. Post-operative rounds by anaesthesiologists after hip fracture surgery: a pilot study. Acta Anaesthesiol Scand 2006;50:437-42.

36. Walke LM, Rosenthal RA, Trentalange M, et al. Restructuring care for older adults undergoing surgery: preliminary data from the
Co-Management of Older Operative Patients En Route Across Treatment Environments (CO-OPERATE) model of care. J Am Geriatr Soc 2014;62:2185-90.

37. Husted H, Jorgensen CC, Gromov K, et al. Low manipulation prevalence following fast-track total knee arthroplasty. Acta Orthop 2015;86:86-91.

38. Khan SK, Malviya A, Muller SD, et al. Reduced short-term complications and mortality following enhanced recovery primary hip and knee arthroplasty: results from 6,000 consecutive procedures. Acta Orthop 2014;85:26-31.

39. Vetter TR, McGwin G Jr. Fast food nation and fast-track arthroplasty: can we have our cake and eat it too? Anesth Analg 2015;120:5-7.

40. Dindo D, Demartines N, Clavien PA. Classification of surgical complications: a new proposal with evaluation in a cohort of 6336 patients and results of a survey. Ann Surg 2004;240:205-13.

41. Slankamenac K, Nederlof N, Pessaux P, et al. The comprehensive complication index: a novel and more sensitive endpoint for assessing outcome and reducing sample size in randomized controlled trials. Ann Surg 2014;260:757-62.

42. Grocott MP, Browne JP, Van der Meulen J, et al The postoperative morbidity survey was validated and used to describe morbidity after major surgery. J Clin Epidemiol 2007;60:919-28.

43. van Walraven $C$, Bennett $C$, Jennings $A$, et al Proportion of hospital readmissions deemed avoidable: a systematic review. CMAJ 2011;183:E391-402. 